Anaesthesist $2021 \cdot 70: 872$

https://doi.org/10.1007/s00101-021-01032-4

Angenommen: 9. August 2021

Online publiziert: 16. September 2021

(c) Springer Medizin Verlag $\mathrm{GmbH}$, ein Teil von

Springer Nature 2021
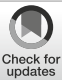

\section{Therapie der aortoösophagealen Fistel unter Berücksichtigung der ESVS-Leitlinie}

\author{
Jan David Süss \\ Klinik für Gefäßchirurgie und Endovaskuläre Chirurgie, St. Antonius Hospital gGmbH Eschweiler, \\ Akademisches Lehrkrankenhaus der RWTH Aachen, Eschweiler, Deutschland
}

\section{Erwiderung}

Grannemann JJ, Röper A, Rehberg S, Janses G (2021) Leitlinien zur Notfalltherapie der aortoösophagealen Fistel sind erforderlich. Anaesthesist. https://doi.org/10.1007/s00101021-01033-3

\section{Originalbeitrag}

Grannemann JJ, Röper A, Rehberg S et al (2021) Die aortoösophageale Fistel - Eine seltene Differenzialdiagnose der oberen Gastrointestinalblutung. Anaesthesist. https:// doi.org/10.1007/s00101-021-00963-2

Sehr geehrte Damen und Herren,

zunächst einmal Gratulation zu der didaktisch guten Kasuistik und Übersicht im oben genannten Artikel.

Der oben genannte Artikel weist einen inhaltlichen Fehler mit der Aussage "Therapeutisch gibt es bislang keine Leitlinienempfehlungen für das Management von AÖF." auf. Diesbezüglich kann die Leitlinie der Europäischen Gesellschaft für Gefäßchirurgie zitiert werden, in der ein gesamtes Kapitel den aortoösophagealen Fisteln gewidmet ist [1]. Unabhängig von der Entität der Fistel kann diese Leitlinie als Leitfaden dienen.

Darüber hinaus sei noch erwähnt, dass die TEVAR nur als reines Bridging-Verfahren angesehen werden kann, denn der thorakale Endostent muss bei persistierendem ösophagealen Defekt als infiziert betrachtet werden. Als kurativer Therapieansatz gelten prinzipiell „immer" bei Patienten, die "fit for surgery" sind, die Fistelsanierung und der thorakale Aortenersatz mit infektresistentem Material (Allograft, Pericardpatch-Tube etc.) in Kombination mit ei- ner Langzeitantibiose. Natürlich adressiert der Artikel primär das Notfallmanagement und nicht die definitive Therapie, aber es soll nach aktuellem Wissensstand nicht der Eindruck entstehen, dass die TEVAR das eigentliche Problem löst.

\section{Korrespondenzadresse}

Dr. med. Jan David Süss

Klinik für Gefäßchirurgie und Endovaskuläre

Chirurgie, St. Antonius Hospital gGmbH Eschweiler, Akademisches Lehrkrankenhaus der RWTH Aachen

Dechant-Deckers-Straße 8, 52249 Eschweiler, Deutschland

jandsuess@yahoo.de

Interessenkonflikt. J.D. Süss gibt an, dass kein Interessenkonflikt besteht.

\section{Literatur}

1. Chakfé N, Diener H, Lejay A et al (2020) Editor's Choice - European Society for Vascular Surgery (ESVS) 2020 Clinical Practice Guidelines on the Management of Vascular Graft and Endograft Infections. European Journal of Vascular and Endovascular Surgery 59:339-384 